\title{
Comparison of Deep Vein Thrombosis Risks in Acute Respiratory Distress Syndrome Caused by COVID-19 and Bacterial Pneumonia: A Retrospective Cohort Study
}

$\mathrm{Na}$ Cui

Beijing Chao-Yang Hospital

Chunguo Jiang

Beijing Chao-Yang Hospital

Chenlu Yang

Chinese Academy of Medical Sciences \& Peking Union Medical College

Liming Zhang

Beijing Chao-Yang Hospital

Xiaokai Feng ( $\sim$ fengxiaokai2020@163.com )

Beijing Chao-Yang Hospital

\section{Research Article}

Keywords: Acute Respiratory Distress Syndrome, Pneumonia, Bacterial, COVID-19, Deep Vein Thrombosis

Posted Date: February 4th, 2022

DOI: https://doi.org/10.21203/rs.3.rs-1250468/v2

License: @ (i) This work is licensed under a Creative Commons Attribution 4.0 International License.

Read Full License 


\section{Abstract}

Background: High incidence of deep vein thrombosis (DVT) has been observed in patients with acute respiratory distress syndrome (ARDS) caused by COVID-19 and those by bacterial pneumonia. However, it is also important to differentiate between these two groups of patients.

Study Design and Methods: We performed a retrospective cohort study to investigate the difference of DVT between the two independent cohorts of ARDS and eventually enrolled 240 patients, 105 of whom with ARDS caused by COVID-19 and 135 by bacterial pneumonia. We analyzed demographics and clinical characteristics for patients with and without DVT in these two cohorts and explored the main differences and similarities between them.

Results: The 28-days incidence of DVT in COVID-19 cohort was higher than that in bacterial pneumonia cohort (57.1\% vs $41.5 \%, P=0.016)$. Taking death as competitive risk, Fine-Gray test showed no significant difference in 28-day cumulative incidence of DVT between these two groups $(P=0.220)$. Fine-Gray competing risk analysis showed an association between CK (creatine kinase isoenzyme)-MB levels, $\mathrm{PaO} 2$ (partial pressure of arterial oxygen)/FiO2 (fraction of inspired oxygen) ratios, D-dimer levels and DVT in COVID-19 cohort and an association between serum creatinine levels, IMV, and DVT in bacterial pneumonia cohort. The sensitivity and specificity of corresponding receiver operating characteristic curve originating from the combination of CK-MB levels, $\mathrm{PaO} 2 / \mathrm{FiO} 2$ ratios and D-dimer levels $\geq 0.5 \mu \mathrm{g} / \mathrm{mL}$ was not inferior to those of the Padua prediction score and the Wells score for screening for DVT in COVID-19 cohort.

Conclusions: Compared with patients with ARDS caused by bacterial pneumonia, the incidence of DVT is higher by logistic model in patients with ARDS caused by COVID-19, and the risk factors for DVT are completely different. Our novel prediction model can aid early identifying patients with high risk for DVT. Keywords: Acute Respiratory Distress Syndrome; Pneumonia, Bacterial; COVID-19; Deep Vein Thrombosis

\section{Introduction}

Deep vein thrombosis (DVT) and pulmonary embolism (PE), collectively referred to as venous thromboembolism (VTE), constitute a major global burden of disease. ${ }^{1}$ Coronavirus disease 2019 (COVID-19), like some other viruses, may also have significant impact on the hematopoietic and hemostatic systems resulting in thrombotic and bleeding complications. ${ }^{2-5}$ Our previous study confirmed that infection of

severe acute respiratory syndrome coronavirus 2 (SARS-CoV-2) might result in an increased risk of VTE, especially the incidence of DVT increased rapidly in proportion to disease severity. ${ }^{6}$ Then in another study we found that the incidence of DVT is extremely high in patients with ARDS and may be associated with adverse outcomes. ${ }^{7}$ 
However, the DVT risks in ARDS have not been compared between patients with COVID-19 and those with bacterial pneumonia. The aim of this study therefore was to compare the incidence and risks of DVT between patients with ARDS caused by COVID-19 and those by bacterial pneumonia, and to further test that the COVID-19 is an additional risk factor and provide some guidance for DVT in ARDS patients infected with COVID-19.8

\section{Methods}

\section{Study Design and Population}

This analysis was a retrospective cohort study. All of the COVID-19 subjects were confirmed by using results of laboratory tests and were hospitalized in West Branch of Union Hospital (affiliated with Tongji Medical College, Huazhong University of Science and Technology), Wuhan, China, one of the major designated referral and treatment hospitals for critically ill adult patients ( $\geq 18$ years old) with COVID-19 from January 29, 2020, to February 29, 2020, in accordance with the World Health Organization's interim guidance. The bacterial pneumonia ARDS cases were from a single-center retrospective cohort study of patients ( $\geq 18$ years old) at Beijing Chao-Yang Hospital, Beijing, China. All of the cases were confirmed by using laboratory test results, and corresponding patients were hospitalized from January 1, 2015, to June 30,2021 . All of the patients met the criteria of the Berlin definition for diagnosis of ARDS. ${ }^{9}$

The exclusion criteria include: active malignant tumor, cerebral stroke, acute myocardial infarction, serious trauma, major operation lasting longer than 45 minutes, fracture of lower limb, and joint replacement for hip or knee. Patients with a survival time less than 3 days and patients without lower extremity venous compression ultrasound data were also excluded.

The first ultrasound examination was performed within 1-3 days after the diagnosis of ARDS, and then the ultrasound examination was reexamined again according to the patient's condition. After intensive treatment, if the patient remained unstable because of conditions such as unexplained hypoxemia or cardiac insufficiency, he or she should be reexamined by ultrasound. If there was more than one ultrasound scan for a single patient, all the results were recorded. Patients were divided into a DVT and a non-DVT group according to the results of the venous compression ultrasound of the lower extremities. The flow chart is shown in Figure $1(A, B)$.

The study was approved by the Union Hospital, affiliated with Tongji Medical College, Huazhong University of Science and Technology (2020-0197) and the ethics committees of the Beijing Chao-Yang Hospital (2020-ke-429), and was conducted in accordance with the 1964 Helsinki Declaration and its later amendments or comparable ethical standards.

\section{Clinical Data}

We analyzed the medical records of the enrolled patients. Data, which included demographic information, clinical history, vital signs, laboratory findings, treatments, complications, and outcomes of the patients 
during hospitalization, were collected and analyzed. We analyzed the survival rates of all patients within 28 days after a diagnosis of ARDS. For patients discharged within 28 days, we followed up by telephone concerning their survival status after discharge.

Ultrasound Assessment

Bedside ultrasound examinations were performed using a portable color ultrasound scanner (CX50, Philips Medical Systems, the Netherlands, equipped with an L12-3/S5-1 probe or EPIQ 7C, Philips Medical Systems, Andover, MA, equipped with an L12-5/S5-1 probe or a Mindray portable Ultrasound M9, GD, China, equipped with an L10-3 probe). The lower extremity venous compression ultrasound was obtained from the institution's Picture Archiving and Communication System. The levels of DVT included the bilateral common femoral, deep and superficial femoral veins, the popliteal veins, and the anterior tibial, posterior tibial, peroneal, and calf muscle veins.

\section{Definitions}

ARDS was defined according to the Berlin definition. ${ }^{9}$ COVID-19 was diagnosed according to the Chinese Management Guideline for COVID-19 (version 6.0). ${ }^{10}$ Bacterial pneumonia, including community-acquired pneumonia and hospital-acquired pneumonia, was diagnosed according to the Clinical Practice Guidelines of the Infectious Diseases Society of America and the America Thoracic Society. ${ }^{11,12}$ A distal thrombosis was defined as a thrombosis in the veins of the calf muscle or in at least 1 branch of the 3 pairs of deep calf veins (anterior tibial vein, posterior tibial vein, or peroneal vein); a proximal thrombosis was defined as a thrombosis in the popliteal vein or above. The Padua prediction score was defined according to the Barbar model. ${ }^{13}$ The Wells score for DVT was defined according to the Di Nisio model ${ }^{1}$. We applied the Acute Physiology and Chronic Health Evaluation (APACHE) $\otimes$ score and the Sequential Organ Failure Assessment (SOFA) score to assess the severity of disease. ${ }^{14,15}$

Statistical Analyses

Categorical variables were described as number and percentage (\%) and continuous variables, as mean, standard deviation, median, and interquartile range. The Shapiro-Wilk test was used to verify normality. Differences between the DVT and the non-DVT groups were assessed by a two-sample $t$-test for normally distributed continuous variables, the Mann-Whitney $U$ test for non-normally distributed continuous variables, and the $\chi^{2}$ or Fisher exact test for categorical variables. We took death as the competitive risk and plotted 28-day cumulative incidence curves (points estimates with 95\% confidence interval [Cl]) for COVID-19 and bacteria ARDS patients, and used Fine-Gray competitive risk model to explore the risk factors of DVT under COVID-19 and bacterial pneumonia subgroups respectively. The adjusted hazards ratio (HR) with $95 \% \mathrm{Cl}$ was reported. To further evaluate the observed differences in risk factors for DVT between COVID-19 and bacterial pneumonia, we utilized interaction terms between ARDS type and each risk factor. A receiver operating characteristic $(\mathrm{ROC})$ analysis was performed to calculate the sensitivity and specificity of risk factors for screening for DVT. The comparison methods of diagnostic accuracy for 
screening for DVT in the ARDS cohort caused by COVID-19 are as follows: Patients were split by generating random numbers to produce a training data set $(n * 0.7)$ and a validation data set $(n * 0.3)$. The area under receiver operating curves (ROC-AUCs) for different risk factors were compared using the method of DeLong et al. ${ }^{16}$ In order to enhance the practicability of the prediction model, we drew a nomogram based on the predictors selected from COVID-19 ARDS population. Calibration was evaluated with calibration plots, which used the bootstrap method of 1000 resampling to show the relationship between the actually observed frequency and the prediction probability through a graph. In a wellcalibrated model, the prediction should fall on a 45-degree diagonal. All statistical analyses were performed using the Statistical Analysis System, version 9.4 (SAS Institute, Cary, NC, USA). All tests were two-tailed; $P<0.05$ was considered statistically significant.

\section{Results}

A total of 240 patients with ARDS were enrolled in this study; 105 patients were considered to belong to the COVID-19 ARDS cohort and 135 patients to the bacterial pneumonia ARDS cohort. The flow chart is shown in Figure 1 ( $A, B)$.

\section{Ultrasound scan for screening for DVT}

Lower extremity venous compression ultrasound scanning was performed whenever feasible for 240 patients regardless of clinical symptoms of the lower limbs (Figure 1B), and the median number of ultrasound scans was 1 (range, 1-5). Eighty (80/240) developed DVT was found and the other 160 was a negative result at the first ultrasound scan. Subsequently, 75 patients underwent more than one ultrasound scans, for whom 36 developed DVT and 39 had no DVT with 2 (range, 2-5) ultrasound examinations. The interval from the diagnosis of ARDS to the occurrence of DVT in the 36 developed DVT group was $8(3,14)$ days, and the interval from the diagnosis of ARDS to the last ultrasound examination in the 39 non-DVT group was $10(5,16)$ days. There was no difference between the two groups ( $P=0.344)$. Finally, of the 240 patients, $116(48.3 \%)$ developed DVT, including 22 with proximal DVT and 94 with distal DVT, 77 of whom had muscular calf vein thrombosis only. The incidence of asymptomatic DVT was 94 (39.2\%) including $15(6.3 \%)$ proximal DVT and 79 (32.9\%) distal DVT, of whom muscular calf vein thrombosis accounted for 67 (27.9\%). For all the 240 patients, the interval from the diagnosis of ARDS to the occurrence of DVT in DVT group was $7(4,12)$ days, and the interval from the diagnosis of ARDS to the last ultrasound examination in non-DVT group was $8(3,14)$ days. There was no difference between the two groups $(P=0.725)$. Six patients were clinically suspected of having $\mathrm{PE} ; 4$ were further confirmed by computed tomography pulmonary angiography (CTPA) examination. (Table 1 and e-Table 1)

\section{Demographic and clinical characteristics of patients in COVID-19 and Bacterial pneumonia ARDS cohorts}

Of the 240 patients with ARDS, 105 were infected with COVID-19 (age [63.6 \pm 13.1$]$ years, male 60 [57.1\%]), 135 with bacterial pneumonia (age [64.8 \pm 15.1$]$ years, male 101 [74.8\%]). Compared with 
patients with bacterial pneumonia, patients with COVID-19 had lesser underlying diseases (smoke,

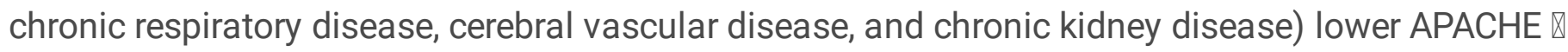
scores and lower SOFA scores $\left(P<0.05\right.$ for all). There was no difference in $\mathrm{PaO}_{2} / \mathrm{FiO}_{2}$ ratios between two groups $(P=0.858)$. More patients with COVID-19 received therapy of glucocorticoids, immunoglobulin, vasoactive drugs and VTE prophylaxis $(P<0.05$ for all). There was significantly higher incidence of DVT (57.1\% vs $41.5 \% ; P=0.016)$ and proximal DVT $(15.2 \%$ vs $4.4 \% ; P=0.004)$ in patients with COVID-19 than those with bacterial pneumonia. (Table 1)

\section{The 28-day cumulative incidence curves of DVT and 28-day cumulative death curves in COVID-19 and Bacterial pneumonia ARDS cohorts}

Took death as competitive risk, the 28-day cumulative incidence rate $(95 \% \mathrm{Cl})$ of DVT in patients with ARDS caused by COVID-19 and by bacterial pneumonia were $85.3 \%(66.6 \%, 92.3 \%)$ and $62.7 \%(48.1 \%$, $72.0 \%)$ respectively. There was no significant difference between the two groups $(P=0.220)$. (Figure 2$)$

\section{Independent predictors of DVT in patients with ARDS caused by COVID-19 and Bacterial Pneumonia}

Took death as competitive risk, we used Fine-Gray competitive risk model to explore the risk factors of DVT under COVID-19 and bacterial pneumonia subgroups respectively (Table 2). Of the 105 ARDS patients with COVID-19, the independent contributors to DVT were CKMB levels (HR, 1.014; $P=0.003$ ), $\mathrm{PaO}_{2} / \mathrm{FiO}_{2}$ ratios ( $\left.\mathrm{HR}, 0.997 ; P=0.079\right)$, and $\mathrm{D}$-dimer levels (HR, 2.975; $\left.P=0.041\right)$, whereas in the bacterial pneumonia ARDS group, DVT was independently associated with serum creatinine levels (HR, $0.951 ; P=0.040)$ and IMV (HR, 2.750; $P=0.004)$. Serum creatinine levels were independently associated with DVT for bacterial pneumonia ARDS patients instead of COVID-19 ARDS patients, nevertheless, the interaction analysis displayed no significant difference between these two groups (test for interaction, $P=$ 0.090; Figure 3). However, the incidence of DVT increased more significantly with rising CK-MB levels in patients with COVID-19 than those with bacterial pneumonia (test for interaction, $P=0.027$; Figure 4).

\section{Comparison of diagnostic accuracy for screening for DVT of different ROCs in ARDS cohort caused by COVID-19}

We propose three new ways of combining forecasting models for screening for DVT based on the significant risk factors in patients with ARDS caused by COVID-19. The sensitivity and specificity of the corresponding ROC curves of the proposed models were not inferior to those of the Padua prediction score and the DVT Well score for screening for DVT (Figure 5 A - C).

\section{Nomogram for screening for DVT of prediction variables}

In order to increase the practicability of the prediction model, we draw a nomogram based on the selected predictors (Figure 6). There are three prediction variables. The corresponding points can be obtained by making a vertical line upward based on the value of each variable. The total points can be obtained by 
adding the points of the three variables. The probability of survival (without DVT) in 5 days, 7 days and 14 days can be obtained by making a vertical line downward based on the total points.

\section{Discussion}

The investigations on the critically ill patients showed that different pathogenic types might account for the high prevalence of DVT. ${ }^{17-19}$ Meanwhile, hypercoagulability appears to be a typical feature of patients with COVID-19. ${ }^{20}$ However, no previous study has compared the DVT risks between the two groups of COVID-19 pneumonia patients and bacterial pneumonia patients. To our knowledge, this study is the first description of DVT difference and hospital mortality in ARDS patients with COVID-19 pneumonia vs Bacterial pneumonia.

In this retrospective cohort study, the 28-days incidence of DVT in the COVID-19 pneumonia patients with ARDS was higher than that in the bacterial pneumonia cohort ( $57.1 \%$ vs $41.5 \%)$. Several reasons probably account for the notably higher incidence of DVT in the COVID-19 pneumonia patients with ARDS. First, it is known that the coagulation pathway can be activated through the contact system and kallikrein/kinin system (KKS). ${ }^{21}$ But it is worth recalling that the KKS is dysregulated by binding of SARS-CoV-2 to the angiotensin-converting enzyme II (ACE-2) receptor of vascular endothelium, this may be a more reasonable mechanism for the noted interaction between COVID-19 and DVT. ${ }^{22,23}$ Second, coronavirus infections may be a trigger for VTE, and several pathogenetic mechanisms, which include endothelial dysfunction, characterized by increased levels of von Willebrand factor; systemic inflammation, by Tolllike receptor activation; and a hypercoagulable state, by tissue factor pathway activation. ${ }^{24}$ Third, high plasma levels of proinflammatory cytokines were observed in the severe COVID-19. ${ }^{25}$ The direct activation of the coagulation cascade by a cytokine storm is conceivable. Lastly, the immune-mediated damage according for the acute coronavirus infections may partially contribute. ${ }^{26}$ Although it is worth noting that after taking death as competitive risk, Fine-Gray test showed no significant difference in the 28-day cumulative incidence of DVT between these two groups $(P=0.220)$. The results showed that the value of the point estimation was different between these two groups. Meanwhile, the confidence intervals were wide. One reason could be the fact that our sample size was small, which may reduce the power of test.

Fine-Gray competing risk analysis in the bacterial pneumonia group showed that serum creatinine levels and IMV were associated with DVT, whereas there was a stronger association between CK-MB levels, $\mathrm{PaO}_{2} / \mathrm{FiO}_{2}$ ratios, D-dimer levels and DVT in COVID-19 group. To further figure out the observed differences in risk factors for DVT between COVID-19 and Bacteria, we utilized interaction terms between COVID status and each risk factor, which suggested that CKMB levels might be independent predictor of DVT in the COVID-19 group compared with bacterial pneumonia.

The incidence of DVT in the COVID-19 pneumonia patients with ARDS increased with the raising of the CK-MB levels. Notwithstanding the incomplete knowledge on its pathophysiology, the mainly suggested mechanisms are: heart and arterial vascular system injury due to increased oxygen demand 
but in the context of hypoxemia triggered by cytokine storm and systemic immune response, which were most frequently encountered among patients with COVID-19 cases. ${ }^{27-32}$ Likewise, it has been hypothesized that a direct viral toxicity through the interaction with ACE-2 receptors highly expressed by some pericytes. ${ }^{29}$ So considering comprehensively above-mentioned factors, it is revealed that severe COVID-19 cases have elevated levels of biomarkers of cardiovascular system injury such as CK-MB. Meanwhile, it is indicated that CK-MB itself might be regarded as a predict marker of DVT. A thorough assessment should therefore be conducted in the follow-up of severe COVID-19 patients with ARDS and adequate measures should be managed to detect, diagnose, and treat VTE at their early stage, considering the high-risk of developing DVT.

We found that serum creatinine may modify the association between the COVID-19 and bacterial pneumonia group and the risk of DVT. The association was stronger among the ARDS patients with bacterial pneumonia. Contrary to findings from our research, some studies have demonstrated that renal impairment is independent risk factor for DVT. ${ }^{33,34}$ It is worth noting that other studies have shown that LWMH may have different levels of bioaccumulation in the case of renal insufficiency. ${ }^{35,36}$ So, we speculate that the same dose of LWMH may play a stronger role in the prevention of DVT because of renal insufficiency. The study by Cook et al. indicated that the incidence of DVT for patients with renal insufficiency in ICU who received dalteparin $5,000 \mathrm{IU}$ once daily was $5.1 \%,{ }^{37}$ which was far lower than that in the overall population of critically ill patients who received prophylaxis recommended by the guidelines. ${ }^{38,39}$ However, we did not find significant association between serum creatinine levels and DVT in patients with COVID-19 pneumonia. There are a number of factors, but one of the biggest reasons is that the patients with COVID-19 had lower serum creatinine levels, lower APACHE $\otimes$ scores and lower SOFA scores compared with those with bacterial pneumonia, the protective effect on DVT of higher levels of serum creatinine was weakened obviously. Unfortunately, due to the retrospective nature of the study, on the one hand, the decrease of LWMH metabolism in patients with AKI and higher level of serum creatinine was based on the conjecture of clinical data analysis, on the other hand, we did not monitor the dynamic change of blood coagulation and detect the activities of plasma levels of coagulation/anticoagulation factors in patients with venous thromboembolism.

Multivariant analysis showed an association only among CK-MB levels, $\mathrm{PaO}_{2} / \mathrm{FiO}_{2}$ ratios, D-dimer levels $\geq 0.5 \mu \mathrm{g} / \mathrm{mL}$, and DVT in COVID-19 cohort. Using a ROC analysis, a combination of the corresponding indicators mentioned above yielded a sensitivity of $66.7 \%$ and a specificity of $82.4 \%$ for prediction for DVT in these hospitalized patients with COVID-19, and the AUC-ROC was 0.804. Statistical test showed that the prediction power of this model was significantly better than DVT Wells score in COVID-19 patients with ARDS. Although there was no significant difference in AUCs between the prediction models, the prediction sensitivity and specificity of the combined model were improved compared with Padua prediction score. This combined prediction model has also been identified to depict effectively for screening for DVT in this group by drawing a nomogram and its calibration curve. A possible reason for the superiority of this new prediction model is that the commonly used predictive scoring systems such as Padua score and Wells score apply to the general medical and surgical patients in hospital. As a 
serious clinical pathophysiological syndrome with an overwhelming inflammatory response and coagulation abnormalities, ARDS caused by COVID-19 has unique clinical characteristics and serious complications.

The prognosis in ARDS patients with COVID-19 pneumonia analyses showed that DVT was associated with adverse outcomes compared with Bacteria ARDS cohort, including length of stay in hospital $(P<$ 0.001). To validate the prognosis of DVT in these two cohorts, we further plotted 28-day cumulative incidence curves of DVT, with death as the competitive risk, and found that the mortality increased with rising incidence of DVT, especially in the COVID-19 cohort. The worse outcome in COVID-19 cohort may be a result of the inflammatory response to SARS-CoV-2 infection resulting in thrombo-inflammation and driving thrombosis. ${ }^{40}$ Coagulation activation could also have been associated with a sustained inflammatory response. ${ }^{41}$ In addition, there is a $50 \%$ chance for patients with untreated proximal DVT to develop symptomatic PE within 3 months. ${ }^{42}$ PE might aggravate the hypoxemia of ARDS patients and then result in lower actuarial survival rates. If there was any clinical suspicion of PE, a CTPA would be considered and obtained, if possible. Unfortunately, due to the critical condition of ARDS patients, CTPA examination was restricted. We only underwent CTPA examination on 1 patient with highly suspected PE and 1 patient was diagnosed with PE in the COVID-19 cohort. By contrast, in the bacterial pneumonia cohort we had performed 5 CTPA examinations, and 3 patients was diagnosed with PE. Using the figures given above, we may significantly underestimate the incidence of PE. The presence of PE associated with DVT may also be a cause of poor survival in patients with DVT. Although these findings are not surprising, given that our patient population represented older, severely ill patients at high risk for DVT, with other organ-related diseases, our data raised the question of screening for DVT, risk stratification, and potential VTE prophylaxis to improve outcomes in ARDS patients infected with COVID19 and those infected with bacterial pneumonia.

This study has some limitations. First, this was a retrospective study that included data from two independent single-center cohorts, which may have resulted in selection bias. Second, our sample size was small, which may underestimate the influence on DVT of factors such as obesity, being bedridden, and the insertion of a central venous catheter. Third, due to the critical condition of patients with ARDS, CTPA examinations were restricted, which significantly underestimated the incidence of PE. Finally, the data from the bacterial pneumonia cohort originated from a 6-year span, whereas the data from the COVID-19 cohort originated from only a 1-month span, which may also have affected the study's results.

\section{Conclusions}

Compared with patients with ARDS caused by bacterial pneumonia, the incidence of DVT is higher by logistic model in patients with ARDS caused by COVID-19, and the risk factors for DVT are completely different. The prediction model based on the combination of CK-MB levels, $\mathrm{PaO}_{2} / \mathrm{FiO}_{2}$ ratios, and $\mathrm{D}$-dimer levels has been identified effectively for screening for DVT in ARDS patients with COVID-19. Future studies investigating the correlation between DVT and COVID-19 should focus on the COVID-19 and its 
implications for thrombosis and anticoagulation, which could provide more experience and evidence regarding COVID-19 treatment measures.

\section{Abbreviations}

ACE-2, angiotensin-converting enzyme II; ALT, alanine aminotransferase; APACHE, Acute Physiology and Chronic Health Evaluation; ARDS, acute respiratory distress syndrome; AST, aspartate aminotransferase; AUC, area under the curve; BUN, blood urea nitrogen; $\mathrm{Cl}$, confidence interval; $\mathrm{CK}$, creatine kinase isoenzyme; COVID-19, coronavirus disease 2019; CTPA, computed tomography pulmonary angiography; $\mathrm{CVC}$, central venous catheterization; DBIL, direct bilirubin; DVT, deep vein thrombosis; $\mathrm{FiO}_{2}$, fraction of inspired oxygen; IMV, invasive mechanical ventilation; IQR, interquartile range; KKS, kallikrein/kinin system; $\mathrm{LDH}$, lactate dehydrogenase; $\mathrm{MV}$, mechanical ventilation; $\mathrm{OR}$, odds ratio; $\mathrm{PaO}_{2}$, partial pressure of arterial oxygen; $\mathrm{PCT}$, procalcitonin; $\mathrm{PE}$, pulmonary embolism; $\mathrm{PT}$, prothrombin time; ROC, receiver operating characteristic; SARS-CoV-2, severe acute respiratory syndrome coronavirus 2; SD, standard deviation; SOFA, Sequential Organ Failure Assessment; TBIL, lower total bilirubin; VTE, venous thromboembolism; WBC, white blood cell.

\section{Declarations}

\section{Acknowledgements}

Not applicable.

\section{Authors' contributions}

NC and CJ designed the study, collected clinical data, analyzed the data, and wrote the manuscript. CY analyzed the data and wrote the manuscript. XF and LZ helped manage the research, performed the statistical analyses, and revised the paper. $\mathrm{NC} \otimes \mathrm{CJ}$ and $\mathrm{CY}$ contributed equally to this article and share first authorship. XF and LZ contributed equally to this article and share corresponding authorship. All authors read and approved the final manuscript.

\section{Funding}

This work was supported by the Research on prevention and control of major chronic noncommunicable diseases of China (Grant NO. 2016YFC1304603 to Dr Xiaokai Feng).

\section{Availability of data and materials}

All data analyzed during the study are presented in the main manuscript. The anonymous dataset is available from the corresponding author upon reasonable request.

\section{Ethics and approval and consent to participate}


This retrospective study involving human participants was approved by the Union Hospital, affiliated with Tongji Medical College, Huazhong University of Science and Technology (2020-0197) and the ethics committee of the Beijing Chao-Yang Hospital, Capital Medical University (2020-ke-429) and was conducted in accordance with the 1964 Helsinki Declaration and its later amendments or comparable ethical standards.

\section{Consent for publication}

Not applicable.

\section{Competing interests}

The authors declare that they have no competing interests.

\section{References}

1. Di Nisio M, van Es N, Büller HR. Deep vein thrombosis and pulmonary embolism.Lancet. 2016;388(10063):3060-3073.

2. Goeijenbier M, van Wissen $M$, van de Weg $C$, et al. Review: Viral infections and mechanisms of thrombosis and bleeding. Journal of medical virology. 2012;84(10):1680-1696.

3. Klok FA, Kruip M, van der Meer NJM, et al. Incidence of thrombotic complications in critically ill ICU patients with COVID-19. Thromb Res. 2020;191:145-147.

4. Terpos E, Ntanasis-Stathopoulos I, Elalamy I, et al. Hematological findings and complications of COVID-19. American journal of hematology. 2020;95(7):834-847.

5. Wang T, Chen R, Liu C, et al. Attention should be paid to venous thromboembolism prophylaxis in the management of COVID-19. The Lancet. Haematology. 2020;7(5):e362-e363.

6. Zhang L, Feng X, Zhang D, et al. Deep Vein Thrombosis in Hospitalized Patients With COVID-19 in Wuhan, China: Prevalence, Risk Factors, and Outcome. Circulation. 2020;142(2):114-128.

7. Cui $\mathrm{N}$, Jiang $\mathrm{C}$, Chen $\mathrm{H}$, Zhang L, Feng $X$. Prevalence, risk, and outcome of deep vein thrombosis in acute respiratory distress syndrome. Thromb J. 2021;19(1):71.

8. Zhang C, Wu Z, Li JW, Zhao H, Wang GQ. Cytokine release syndrome in severe COVID-19: interleukin6 receptor antagonist tocilizumab may be the key to reduce mortality. Int $J$ Antimicrob Agents. 2020;55(5):105954.

9. Ranieri VM, Rubenfeld GD, Thompson BT, et al. Acute respiratory distress syndrome: the Berlin Definition. Jama. 2012;307(23):2526-2533.

10. National Health Commission of the People's Republic of China. Chinese management guideline for COVID-19, version 6.0. http://www.nhc.gov. cn/yzygj/s7653p/202002/8334a8326dd94d329df351d7da8aefc2/files/b2

11. Kalil AC, Metersky ML, Klompas M, et al. Management of Adults With Hospital-acquired and Ventilator-associated Pneumonia: 2016 Clinical Practice Guidelines by the Infectious Diseases 
Society of America and the American Thoracic Society. Clin Infect Dis. 2016;63(5):e61-e111.

12. Metlay JP, Waterer GW, Long AC, et al. Diagnosis and Treatment of Adults with Community-acquired Pneumonia. An Official Clinical Practice Guideline of the American Thoracic Society and Infectious Diseases Society of America. Am J Respir Crit Care Med. 2019;200(7):e45-e67.

13. Barbar S, Noventa F, Rossetto V, et al. A risk assessment model for the identification of hospitalized medical patients at risk for venous thromboembolism: the Padua Prediction Score. J Thromb Haemost. 2010;8(11):2450-2457.

14. Knaus WA, Draper EA, Wagner DP, Zimmerman JE. APACHE II: a severity of disease classification system. Crit Care Med. 1985;13(10):818-829.

15. Lambden S, Laterre PF, Levy MM, Francois B. The SOFA score-development, utility and challenges of accurate assessment in clinical trials. Crit Care. 2019;23(1):374.

16. DeLong ER, DeLong DM, Clarke-Pearson DL. Comparing the areas under two or more correlated receiver operating characteristic curves: a nonparametric approach. Biometrics. 1988;44(3):837-845.

17. Obi AT, Tignanelli CJ, Jacobs BN, et al. Empirical systemic anticoagulation is associated with decreased venous thromboembolism in critically ill influenza A H1N1 acute respiratory distress syndrome patients. J Vasc Surg Venous Lymphat Disord. 2019;7(3):317-324.

18. Cui N, Mi S, Jiang C, et al. Deep vein thrombosis in acute respiratory distress syndrome caused by bacterial pneumonia. BMC Pulm Med. 2021;21(1):264.

19. Ren B, Yan F, Deng Z, et al. Extremely High Incidence of Lower Extremity Deep Venous Thrombosis in 48 Patients With Severe COVID-19 in Wuhan. Circulation. 2020;142(2):181-183.

20. Hezi-Yamit A, Wong PW, Bien-Ly N, et al. Synergistic induction of tissue factor by coagulation factor Xa and TNF: evidence for involvement of negative regulatory signaling cascades. Proceedings of the National Academy of Sciences of the United States of America. 2005;102(34):12077-12082.

21. Schmaier AH. The contact activation and kallikrein/kinin systems: pathophysiologic and physiologic activities. J Thromb Haemost. 2016;14(1):28-39.

22. Shi S, Qin M, Shen B, et al. Association of Cardiac Injury With Mortality in Hospitalized Patients With COVID-19 in Wuhan, China. JAMA cardiology. 2020;5(7):802-810.

23. Yang C, Jin Z. An Acute Respiratory Infection Runs Into the Most Common Noncommunicable Epidemic-COVID-19 and Cardiovascular Diseases. JAMA cardiology. 2020;5(7):743-744.

24. Giannis D, Ziogas IA, Gianni P. Coagulation disorders in coronavirus infected patients: COVID-19, SARS-CoV-1, MERS-CoV and lessons from the past. Journal of clinical virology : the official publication of the Pan American Society for Clinical Virology. 2020;127:104362.

25. Li H, Liu L, Zhang D, et al. SARS-CoV-2 and viral sepsis: observations and hypotheses. Lancet (London, England). 2020;395(10235):1517-1520.

26. Zhang $\mathrm{H}$, Zhou $\mathrm{P}$, Wei $\mathrm{Y}$, et al. Histopathologic Changes and SARS-CoV-2 Immunostaining in the Lung of a Patient With COVID-19. Ann Intern Med. 2020;172(9):629-632.

27. Akhmerov A, Marbán E. COVID-19 and the Heart. Circ Res. 2020;126(10):1443-1455. 
28. Atri D, Siddiqi HK, Lang JP, Nauffal V, Morrow DA, Bohula EA. COVID-19 for the Cardiologist: Basic Virology, Epidemiology, Cardiac Manifestations, and Potential Therapeutic Strategies. JACC. Basic to translational science. 2020;5(5):518-536.

29. Chen L, Li X, Chen M, Feng Y, Xiong C. The ACE2 expression in human heart indicates new potential mechanism of heart injury among patients infected with SARS-CoV-2. Cardiovascular research. 2020;116(6):1097-1100.

30. Zheng YY, Ma YT, Zhang JY, Xie X. COVID-19 and the cardiovascular system. Nature reviews. Cardiology. 2020;17(5):259-260.

31. Zeng JH, Liu YX, Yuan J, et al. First case of COVID-19 complicated with fulminant myocarditis: a case report and insights. Infection. 2020;48(5):773-777.

32. Ammirati E, Wang DW. SARS-CoV-2 inflames the heart. The importance of awareness of myocardial injury in COVID-19 patients. International journal of cardiology. 2020;311:122-123.

33. Kuo TH, Li HY, Lin SH. Acute kidney injury and risk of deep vein thrombosis and pulmonary embolism in Taiwan: A nationwide retrospective cohort study. Thromb Res. 2017;151:29-35.

34. Christiansen CF, Schmidt M, Lamberg AL, et al. Kidney disease and risk of venous thromboembolism: a nationwide population-based case-control study. J Thromb Haemost. 2014;12(9):1449-1454.

35. Mahé $\mathrm{l}$, Aghassarian $\mathrm{M}$, Drouet $\mathrm{L}$, et al. Tinzaparin and enoxaparin given at prophylactic dose for eight days in medical elderly patients with impaired renal function: a comparative pharmacokinetic study. Thromb Haemost. 2007;97(4):581-586.

36. Schmid P, Brodmann D, Odermatt Y, Fischer AG, Wuillemin WA. Study of bioaccumulation of dalteparin at a therapeutic dose in patients with renal insufficiency. $J$ Thromb Haemost. 2009;7(10):1629-1632.

37. Cook D, Douketis J, Meade M, et al. Venous thromboembolism and bleeding in critically ill patients with severe renal insufficiency receiving dalteparin thromboprophylaxis: prevalence, incidence and risk factors. Crit Care. 2008;12(2):R32.

38. Gibson CD, Colvin MO, Park MJ, et al. Prevalence and Predictors of Deep Vein Thrombosis in Critically III Medical Patients Who Underwent Diagnostic Duplex Ultrasonography. J Intensive Care Med. 2020;35(10):1062-1066.

39. Kaplan D, Casper TC, Elliott CG, et al. VTE Incidence and Risk Factors in Patients With Severe Sepsis and Septic Shock. Chest. 2015;148(5):1224-1230.

40. Connors JM, Levy JH. COVID-19 and its implications for thrombosis and anticoagulation. Blood. 2020;135(23):2033-2040.

41. Beristain-Covarrubias N, Perez-Toledo M, Thomas MR, Henderson IR, Watson SP, Cunningham AF. Understanding Infection-Induced Thrombosis: Lessons Learned From Animal Models. Frontiers in immunology. 2019;10:2569.

42. Moheimani F, Jackson DE. Venous thromboembolism: classification, risk factors, diagnosis, and management. ISRN Hematol. 2011;2011:124610. 


\section{Tables}

Table 1 Demographic and clinical characteristics of patients with ARDS caused by COVID-19 and Bacterial pneumonia 


\begin{tabular}{|c|c|c|c|c|}
\hline Characteristic & $\begin{array}{l}\text { Total } \\
(\mathrm{N}=240)\end{array}$ & $\begin{array}{l}\text { Bacterial } \\
\text { pneumonia } \\
(\mathrm{N}=135)\end{array}$ & $\begin{array}{l}\text { COVID-19 } \\
(N=105)\end{array}$ & $\begin{array}{l}P \\
\text { value }\end{array}$ \\
\hline Age, y & $64.3 \pm 14.2$ & $64.8 \pm 15.1$ & $63.6 \pm 13.1$ & 0.522 \\
\hline Male & $161(67.1)$ & $101(74.8)$ & $60(57.1)$ & 0.004 \\
\hline $\mathrm{BMI}, \mathrm{kg} / \mathrm{m}^{2}$ & $23.6 \pm 3.4$ & $23.7 \pm 3.9$ & $23.6 \pm 2.7$ & 0.977 \\
\hline Bed time $\geq 3$ days & 189 (78.8) & $117(86.7)$ & $72(68.6)$ & 0.001 \\
\hline Hospital stays, $d$ & $23(13,38)$ & $18(11,29)$ & $31(18,41)$ & $\begin{array}{l}< \\
0.001\end{array}$ \\
\hline $\begin{array}{l}\text { ARDS to DVT or last US } \\
\text { scan, } d\end{array}$ & $7(3,13)$ & $5(2,12)$ & $10(6,14)$ & $<.001$ \\
\hline $\begin{array}{l}\text { Median number of US } \\
\text { scans }\end{array}$ & $1(1,2)$ & $1(1,2)$ & $1(1,1)$ & $\begin{array}{l}< \\
0.001\end{array}$ \\
\hline DVT Wells score & $1(1,2)$ & $1(1,1)$ & $1(0,2)$ & 0.004 \\
\hline Padua prediction score & $5(5,6)$ & $5(5,6)$ & $5(4,6)$ & 0.171 \\
\hline APACHE II score & $16(12,23)$ & $22(17,27)$ & $11(11,13)$ & $<0.001$ \\
\hline SOFA score & $5(4,10)$ & $6(4,10)$ & $4(3,12)$ & 0.012 \\
\hline \multicolumn{5}{|l|}{ Underlying disease } \\
\hline Smoke & $84(35.0)$ & $76(56.3)$ & $8(7.6)$ & $\begin{array}{l}< \\
0.001\end{array}$ \\
\hline $\begin{array}{l}\text { Chronic respiratory } \\
\text { disease }\end{array}$ & $32(13.3)$ & $25(18.5)$ & $7(6.7)$ & 0.007 \\
\hline Hypertension & 103 (42.9) & $60(44.4)$ & $43(41.0)$ & 0.588 \\
\hline Coronary heart disease & $39(16.3)$ & $24(17.8)$ & 15 (14.3) & 0.467 \\
\hline Diabetes & $54(22.5)$ & $33(24.4)$ & $21(20.0)$ & 0.413 \\
\hline Cerebral vascular disease & $31(12.9)$ & $27(20.0)$ & $4(3.8)$ & $<0.001$ \\
\hline Chronic liver disease & $5(2.1)$ & $2(1.5)$ & $3(2.9)$ & 0.656 \\
\hline Chronic kidney disease & $16(6.7)$ & $13(9.6)$ & $3(2.9)$ & 0.037 \\
\hline \multicolumn{5}{|l|}{ Symptoms of onset } \\
\hline Fever & $222(92.5)$ & $128(94.8)$ & $94(89.5)$ & 0.123 \\
\hline Cough & $188(78.3)$ & $114(84.4)$ & $74(70.5)$ & 0.009 \\
\hline
\end{tabular}




\begin{tabular}{|c|c|c|c|c|}
\hline Dyspnea & $198(82.5)$ & $130(96.3)$ & $68(64.8)$ & $\begin{array}{l}< \\
0.001\end{array}$ \\
\hline DVT symptoms & $46(19.2)$ & $27(20.0)$ & $19(18.1)$ & 0.710 \\
\hline Edema of lower extremities & $42(17.5)$ & $27(20.0)$ & $15(14.3)$ & 0.248 \\
\hline Leg pain & $6(2.5)$ & $2(1.5)$ & $4(3.8)$ & 0.408 \\
\hline \multicolumn{5}{|l|}{ Arterial blood gas analysis } \\
\hline $\mathrm{PaO}_{2} / \mathrm{FiO}_{2}, \mathrm{~mm} \mathrm{Hg}$ & $135(81,195)$ & $137(80,188)$ & $130(81,197)$ & 0.858 \\
\hline \multicolumn{5}{|c|}{ Hematologic and infection-related indices } \\
\hline $\begin{array}{l}\text { White blood cell count, } \\
\times 10^{9} / \mathrm{L}\end{array}$ & $10.9(7.23,16.0)$ & $14.4(10.1,19.0)$ & $8.1(5.7,10.9)$ & $\begin{array}{l}<.001 \\
0.00\end{array}$ \\
\hline Neutrophil count, $\times 10^{9} / \mathrm{L}$ & $9.7(5.9,14.2)$ & $12.8(9.0,17.3)$ & $6.5(4.2,9.6)$ & $\begin{array}{l}<.001 \\
0.00\end{array}$ \\
\hline Lymphocyte count, $\times 10^{9} / \mathrm{L}$ & $0.8(0.5,1.2)$ & $0.8(0.5,1.2)$ & $0.8(0.5,1.1)$ & 0.528 \\
\hline $\begin{array}{l}\text { Neutrophil-to-lymphocyte } \\
\text { ratio }\end{array}$ & $12.5(7.1,21.0)$ & $15.1(8.7,23.7)$ & $8.9(4.7,15.1)$ & $<.001$ \\
\hline Platelet count, $\times 10^{9} / \mathrm{L}$ & $190(133,263)$ & $185(115,261)$ & $190(144,270)$ & 0.205 \\
\hline Hemoglobin, g/L & $116(99,130)$ & $112(87,130)$ & $118(108,132)$ & 0.021 \\
\hline C-reactive protein, $\mathrm{mg} / \mathrm{L}$ & $99.5(51.8,120.0)$ & $120.0(82.0,120.0)$ & $58.0(20.7,99.5)$ & $\begin{array}{l}< \\
0.001\end{array}$ \\
\hline Serum procalcitonin, ng/L & $0.8(0.1,4.2)$ & $3.2(1.2,11.3)$ & $0.1(0.1,0.4)$ & $\begin{array}{l}< \\
0.001\end{array}$ \\
\hline \multicolumn{5}{|l|}{ Biochemical test } \\
\hline Total protein, g/L & $57.6(50.9,63.2)$ & $53.0(47.0,59.0)$ & $60.8(57.6,65.3)$ & $\begin{array}{l}< \\
0.001\end{array}$ \\
\hline Albumin, g/L & $26.3(23.6,29.9)$ & $25.3(23.0,29.6)$ & $27.3(24.2,30.2)$ & 0.007 \\
\hline $\begin{array}{l}\text { Aspartate } \\
\text { aminotransferase, }\end{array}$ & $35.0(25.5,58.0)$ & $40.8(26.0,71.7)$ & $33.0(24.0,44.0)$ & 0.008 \\
\hline \multicolumn{5}{|l|}{$\mathrm{U} / \mathrm{L}$} \\
\hline $\begin{array}{l}\text { Alanine aminotransferase, } \\
\text { U/L }\end{array}$ & $33.2(19.6,60.2)$ & $29.7(17.9,58.8)$ & $35.0(26.0,62.0)$ & 0.101 \\
\hline Total bilirubin, $\mu \mathrm{mol} / \mathrm{L}$ & $14.2(10.1,20.3)$ & $15.2(10.7,22.8)$ & $13.6(9.2,17.0)$ & 0.006 \\
\hline Direct bilirubin, $\mu \mathrm{mol} / \mathrm{L}$ & $4.8(3.1,7.3)$ & $5.2(3.3,8.4)$ & $4.5(3.0,6.2)$ & 0.038 \\
\hline
\end{tabular}




\begin{tabular}{|c|c|c|c|c|}
\hline $\begin{array}{l}\text { Lactate dehydrogenase, } \\
\text { U/L }\end{array}$ & $\begin{array}{l}354.0(234.3 \\
546.0)\end{array}$ & $\begin{array}{l}350.0(239.0 \\
624.8)\end{array}$ & $\begin{array}{l}354.0(224.0 \\
511.0)\end{array}$ & 0.360 \\
\hline $\begin{array}{l}\text { Blood urea nitrogen, } \\
\mathrm{mmol} / \mathrm{L}\end{array}$ & $7.54(4.80,13.78)$ & $\begin{array}{l}10.21(5.39 \\
17.42)\end{array}$ & $6.50(4.21,9.21)$ & $\begin{array}{l}< \\
0.001\end{array}$ \\
\hline Serum creatinine, $\mu \mathrm{mol} / \mathrm{L}$ & $73.5(56.7,125.8)$ & $89.1(62.4,193.0)$ & $64.3(53.7,75.5)$ & $\begin{array}{l}<.001 \\
0.001\end{array}$ \\
\hline CK-MB, U/L & $16.2(10.8,29.7)$ & $16.2(11.0,26.9)$ & $16.2(10.0,31.0)$ & 0.812 \\
\hline \multicolumn{5}{|l|}{ Coagulation function } \\
\hline D-dimer, $\mu \mathrm{g} / \mathrm{mL}$ & $1.8(0.7,4.6)$ & $1.5(0.6,2.6)$ & $2.8(1.1,8.0)$ & $<.001$ \\
\hline Prothrombin time, s & $13.6(12.60,15.1)$ & $13.5(12.3,15.2)$ & $13.6(12.7,14.9)$ & 0.193 \\
\hline $\begin{array}{l}\text { Activated partial } \\
\text { thromboplastin time, s }\end{array}$ & $33.7(29.7,38.1)$ & $32.1(28.7,35.7)$ & $34.8(32.5,39.2)$ & $\dot{0.001}$ \\
\hline DVT & $116(48.3)$ & $56(41.5)$ & $60(57.1)$ & 0.016 \\
\hline Proximal DVT & $22(9.2)$ & $6(4.4)$ & $16(15.2)$ & 0.004 \\
\hline Distal DVT & $94(39.2)$ & $50(37.0)$ & $44(41.9)$ & 0.443 \\
\hline $\begin{array}{l}\text { Muscular calf vein } \\
\text { thrombosis only }\end{array}$ & $77(32.1)$ & $38(28.1)$ & $39(37.1)$ & 0.139 \\
\hline \multicolumn{5}{|l|}{ Treatments } \\
\hline Glucocorticoid therapy & $90(37.5)$ & $40(29.6)$ & $50(47.6)$ & 0.004 \\
\hline Immunoglobulin therapy & $56(23.3)$ & $3(2.2)$ & $53(50.5)$ & $\begin{array}{l}< \\
0.001\end{array}$ \\
\hline CVC & $82(34.2)$ & $45(33.3)$ & $37(35.2)$ & 0.758 \\
\hline CRRT & $22(9.2)$ & $12(8.9)$ & $10(9.5)$ & 0.866 \\
\hline IMV & $103(42.9)$ & 79 (58.5) & $24(22.9)$ & $\dot{<} 001$ \\
\hline Sedative therapy & $86(35.8)$ & $62(45.9)$ & $24(22.9)$ & $\dot{0} 001$ \\
\hline Vasoactive drugs & $64(26.7)$ & $27(20.0)$ & $37(35.2)$ & 0.008 \\
\hline VTE prophylaxis & $137(57.1)$ & $64(47.4)$ & $73(69.5)$ & 0.001 \\
\hline LMWH & $117(48.8)$ & 55 (40.7) & $62(59.0)$ & 0.005 \\
\hline LMWH + physical & $80(33.3)$ & $40(29.6)$ & $40(38.1)$ & 0.168 \\
\hline Physical prophylaxis only & $21(8.8)$ & $8(5.9)$ & $13(12.4)$ & 0.079 \\
\hline
\end{tabular}


Data are presented as mean \pm SD, median (IQR), or $n(\%)$. $P$ values comparing DVT and non-DVT groups were from a two-sample $t$-test, Mann-Whitney $U$ test, $\chi^{2}$ test, or Fisher exact test. $P<0.05$ was considered statistically significant.

Abbreviations: APACHE, Acute Physiology and Chronic Health Evaluation; ARDS, acute respiratory distress syndrome; $\mathrm{BMI}$, body mass index; $\mathrm{CK}$, creatine kinase isoenzyme; COVID-19, coronavirus disease 2019; CRRT, continuous renal replacement therapy; CVC, central venous catheterization; DVT, deep venous thrombosis; $\mathrm{FiO}_{2}$, fraction of inspired oxygen; IMV, invasive mechanical ventilation; IQR, interquartile range; $\mathrm{LMWH}$, low molecular weight heparin; $\mathrm{PaO}_{2}$, partial pressure of arterial oxygen; $\mathrm{PE}$, pulmonary embolism; SD, standard deviation; SOFA, Sequential Organ Failure Assessment; US, ultrasound; VTE, venous thromboembolism.

Table 2 Predictors of DVT in patients with ARDS caused by COVID-19 and Bacterial pneumonia 


\begin{tabular}{|c|c|c|c|c|c|c|c|}
\hline \multirow[t]{3}{*}{ Variable } & \multicolumn{2}{|c|}{$\begin{array}{l}\text { Total ARDS } \\
(\mathrm{N}=240)\end{array}$} & \multicolumn{2}{|c|}{$\begin{array}{l}\text { Bacterial } \\
\text { Pneumonia } \\
(\mathrm{N}=135)\end{array}$} & \multicolumn{2}{|l|}{$\begin{array}{l}\text { COVID-19 } \\
(\mathrm{N}=105)\end{array}$} & \multirow[t]{3}{*}{$\begin{array}{l}P \text { for } \\
\text { Interaction } \\
\text { With COVID- } \\
19 \text { Status }\end{array}$} \\
\hline & $\begin{array}{l}\text { Adjusted } \\
\text { HR }\end{array}$ & $\begin{array}{l}P \\
\text { value }\end{array}$ & $\begin{array}{l}\text { Adjusted } \\
\text { HR }\end{array}$ & $\begin{array}{l}P \\
\text { value }\end{array}$ & $\begin{array}{l}\text { Adjusted } \\
\text { HR }\end{array}$ & $\begin{array}{l}P \\
\text { value }\end{array}$ & \\
\hline & $(95 \% \mathrm{Cl})$ & & $(95 \% \mathrm{Cl})$ & & $(95 \% \mathrm{Cl})$ & & \\
\hline Age, & 1.165 & 0.028 & 1.217 & 0.061 & 1.015 & 0.878 & \\
\hline per 10 years & $\begin{array}{l}\text { (1.017, } \\
1.334)\end{array}$ & & $\begin{array}{l}(0.991, \\
1.494)\end{array}$ & & $\begin{array}{l}(0.834, \\
1.236)\end{array}$ & & \\
\hline \multirow{2}{*}{$\begin{array}{l}\text { Serum creatinine, } \\
\text { per } 10 \mu \mathrm{mol} / \mathrm{L}\end{array}$} & 0.955 & 0.006 & 0.951 & 0.040 & 0.988 & 0.288 & 0.090 \\
\hline & $\begin{array}{l}(0.924, \\
0.987)\end{array}$ & & $\begin{array}{l}(0.907 \\
0.998)\end{array}$ & & $\begin{array}{l}(0.966, \\
1.010)\end{array}$ & & \\
\hline \multirow{2}{*}{$\begin{array}{l}\text { Serum } \\
\text { procalcitonin, } \\
\text { per } 1 \mathrm{ng} / \mathrm{L}\end{array}$} & 1.001 & 0.957 & 1.005 & 0.734 & 1.316 & 0.266 & \\
\hline & $\begin{array}{l}(0.977 \\
1.025)^{\prime}\end{array}$ & & $\begin{array}{l}(0.975, \\
1.037)\end{array}$ & & $\begin{array}{l}(0.811, \\
2.134)\end{array}$ & & \\
\hline \multirow{2}{*}{$\begin{array}{l}\text { CK-MB, } \\
\text { per } 1 \mathrm{U} / \mathrm{L}\end{array}$} & 0.998 & 0.328 & 0.994 & 0.214 & 1.014 & 0.003 & 0.027 \\
\hline & $\begin{array}{l}(0.994 \\
1.002)\end{array}$ & & $\begin{array}{l}(0.984, \\
1.004)\end{array}$ & & $\begin{array}{l}(1.005, \\
1.024)\end{array}$ & & \\
\hline \multirow{2}{*}{$\begin{array}{l}\mathrm{PaO}_{2} / \mathrm{FiO}_{2} \\
\text { per } 1 \mathrm{mmHg}\end{array}$} & 0.996 & 0.012 & 0.996 & 0.166 & 0.997 & 0.079 & \\
\hline & $\begin{array}{l}(0.993 \\
0.999)\end{array}$ & & $\begin{array}{l}(0.991, \\
1.002)\end{array}$ & & $\begin{array}{l}(0.993, \\
1.000)\end{array}$ & & \\
\hline \multicolumn{8}{|l|}{ D-dimer } \\
\hline$<0.5 \mu \mathrm{g} / \mathrm{mL}$ & Reference & & Reference & & & & \\
\hline \multirow[t]{2}{*}{$\geq 0.5 \mu \mathrm{g} / \mathrm{mL}$} & 1.911 & 0.011 & 1.534 & 0.225 & 2.975 & 0.041 & \\
\hline & $\begin{array}{l}(1.163, \\
3.139)\end{array}$ & & $\begin{array}{l}(0.768, \\
3.063)\end{array}$ & & $\begin{array}{l}(1.046, \\
8.464)\end{array}$ & & \\
\hline \multicolumn{8}{|l|}{ IMV } \\
\hline No & Reference & & Reference & & & & \\
\hline \multirow[t]{2}{*}{ Yes } & 1.676 & 0.009 & 2.750 & 0.004 & 0.823 & 0.524 & \\
\hline & $\begin{array}{l}(1.140, \\
2.464)\end{array}$ & & $\begin{array}{l}(1.389, \\
5.443)\end{array}$ & & $\begin{array}{l}(0.453, \\
1.497)\end{array}$ & & \\
\hline
\end{tabular}

Fine and Gray competing risk analysis was performed in the ARDS cohorts. The interactions of ARDS type (COVID-19 status) with age, serum creatinine level, serum procalcitonin level, CK-MB level, $\mathrm{PaO}_{2} / \mathrm{FiO}_{2}$, D-dimer level, and IMV were included in the analysis. 
Abbreviations: ARDS, acute respiratory distress syndrome; CK, creatine kinase isoenzyme;

$\mathrm{Cl}$, confidence interval; COVID-19, coronavirus disease 2019; DVT, deep venous thrombosis; $\mathrm{FiO}_{2}$, fraction of inspired oxygen; IMV, invasive mechanical ventilation; $\mathrm{OR}$, odds ratio; $\mathrm{PaO}_{2}$, partial pressure of arterial oxygen.

\section{Figures}
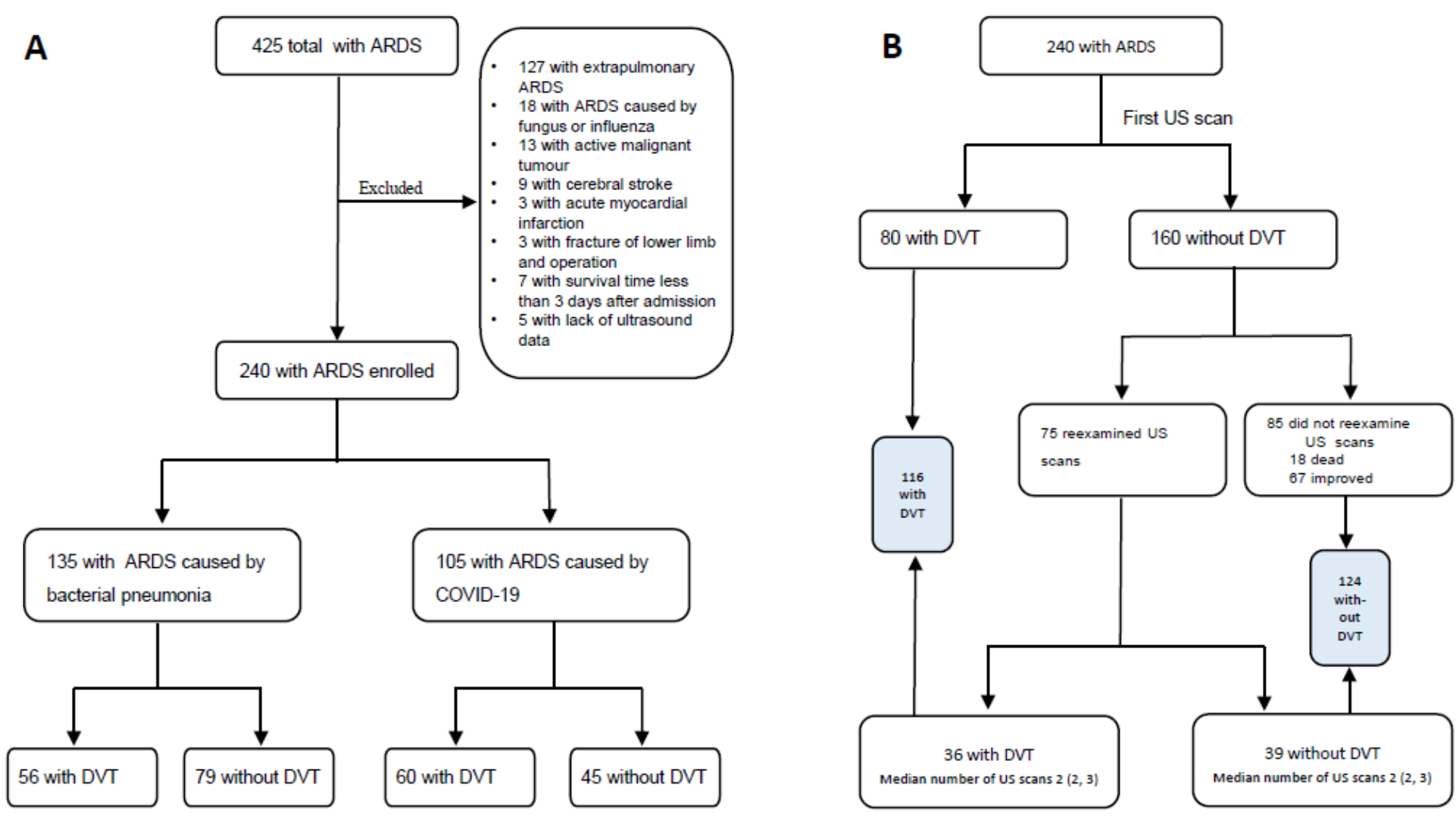

Figure 1

(A, B) Study flow chart.

A, flow chart for including patients; B, flow chart for screening for DVT.

The interval from the diagnosis of ARDS to the occurrence of DVT in the DVT group was $7(4,12)$ days, and the interval from the diagnosis of ARDS to the last ultrasound examination in the non-DVT group was $8(3,14)$ days. There were no differences between the two groups $(P=0.725)$.

Abbreviations: ARDS, acute respiratory distress syndrome; COVID-19, coronavirus disease 2019; DVT, deep vein thrombosis; US, ultrasound 


\section{Cumulative incidence functions}

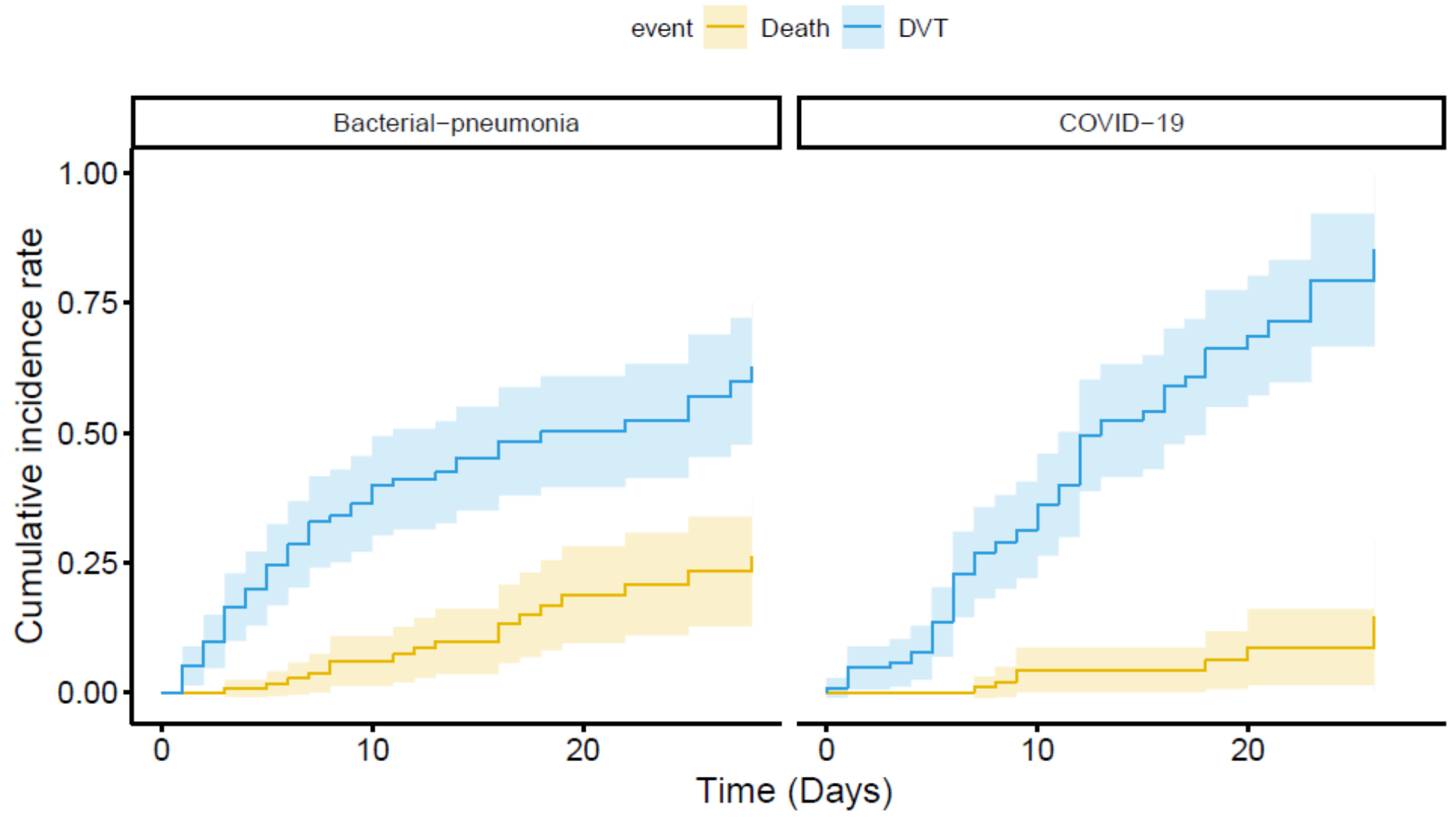

Figure 2

The 28-day cumulative incidence curves of DVT and 28-day cumulative death curves in COVID-19 and Bacterial pneumonia ARDS cohorts.

Took death as the competitive risk, Fine-Gray test showed no significant difference in the 28-day cumulative prevalence of DVT between COVID-19 ARDS and Bacterial pneumonia ARDS group $(P=$ $0.220)$.

Abbreviations: ARDS, acute respiratory distress syndrome; COVID-19, coronavirus disease 2019; DVT, deep vein thrombosis.

\section{Figure 3}

Prevalence of DVT decreased with serum creatinine levels in the Bacterial pneumonia ARDS group.

Serum creatinine levels were independently associated with DVT for Bacterial pneumonia ARDS patients (red line) instead of COVID-19 ARDS patients (green line), nevertheless, the interaction analysis displayed no significant difference between two groups (test for interaction, $P=0.090$ ). Data are adjusted for age, serum PCT levels, CK-MB levels, D-dimer levels, $\mathrm{PaO}_{2} / \mathrm{FiO}_{2}$ ratios, and IMV. 
Abbreviations: ARDS, acute respiratory distress syndrome; CK, creatine kinase isoenzyme; COVID-19, coronavirus disease 2019; DVT, deep vein thrombosis; $\mathrm{FiO}_{2}$, fraction of inspired oxygen; IMV, invasive mechanical ventilation; $\mathrm{PaO}_{2}$, partial pressure of arterial oxygen; $\mathrm{PCT}$, procalcitonin

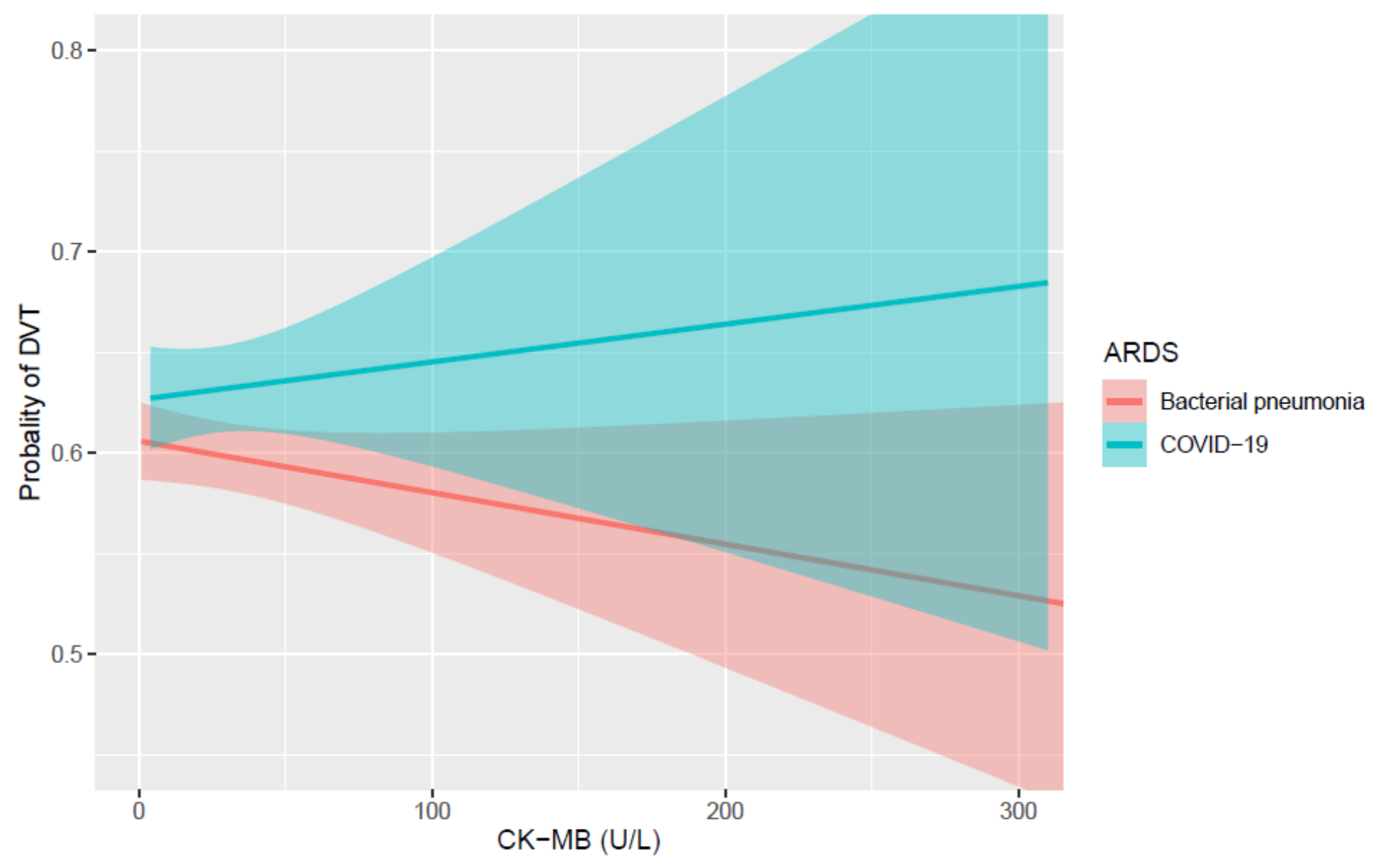

\section{Figure 4}

Prevalence of DVT increased with CK-MB levels only in the COVID-19 ARDS group.

The occurrence of DVT in COVID-19 ARDS group (green line) increased with rising CK-MB levels, whereas there was no association between DVT and CK-MB levels in Bacterial pneumonia ARDS group (red line; test for interaction, $P=0.027$ ). Data are adjusted for age, serum creatinine levels, serum PCT levels, $D$ dimer levels, $\mathrm{PaO}_{2} / \mathrm{FiO}_{2}$ ratios, and IMV.

Abbreviations: ARDS, acute respiratory distress syndrome; CK, creatine kinase isoenzyme; COVID-19, coronavirus disease 2019; DVT, deep vein thrombosis; $\mathrm{FiO}_{2}$, fraction of inspired oxygen; IMV, invasive mechanical ventilation; $\mathrm{PaO}_{2}$, partial pressure of arterial oxygen; $\mathrm{PCT}$, procalcitonin 
A
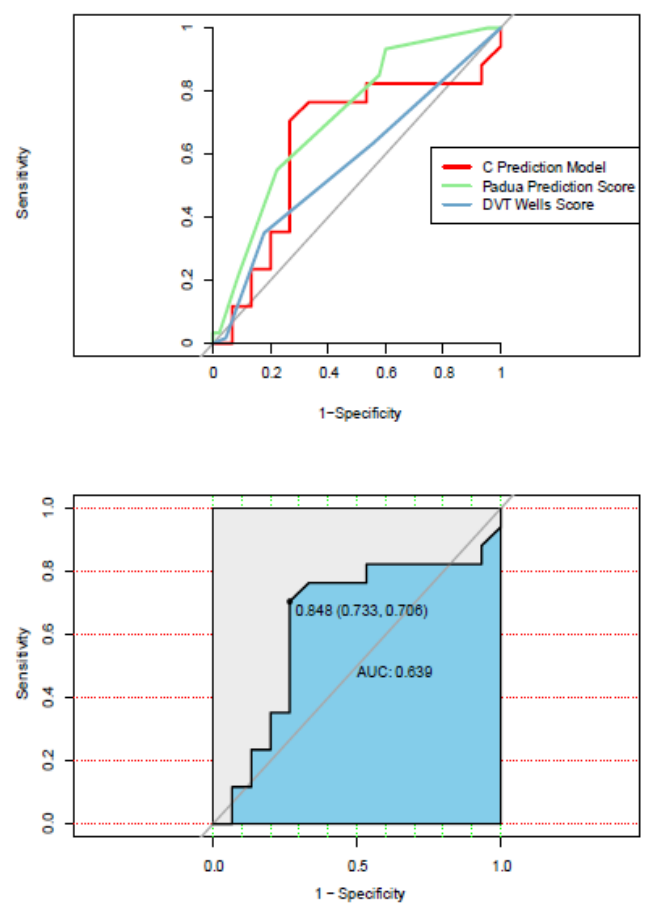

B

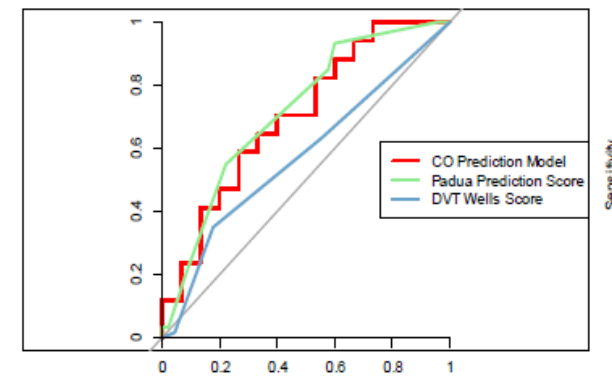

1-Specificity

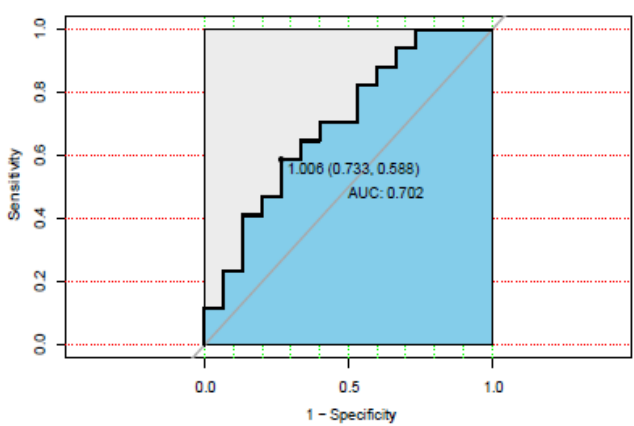

C
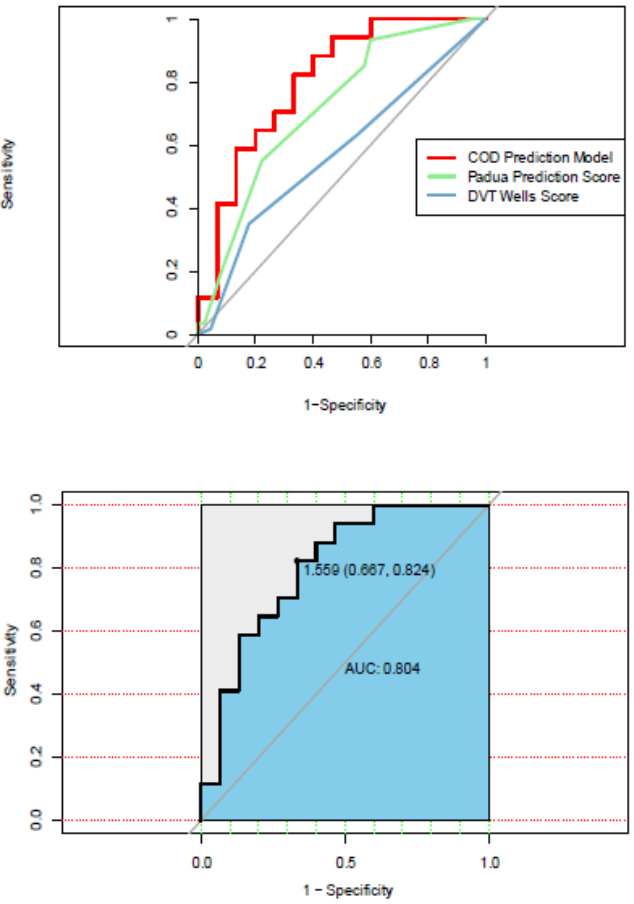

Figure 5

(A-C) Comparison of diagnostic accuracy for screening for DVT of different ROCs in ARDS cohort caused by COVID-19.

Patients were split by generating random numbers to produce a training data set $(n \star 0.7)$ and a validation data set $(n * 0.3)$ in ARDS cohort caused by COVID-19.

A, the CK-MB level shows satisfactory forecasting ability for DVT (AUC $=0.639 ; 95 \%$ Cl: $0.428-0.850$; sensitivity: 70.6\%; specificity: $73.3 \%$ ) which has no significant difference compared with the DVT Wells score (AUC $=0.537 ; P=0.587$ for these two curves) and the Padua prediction score (AUC $=0.717 ; P=$ 0.515 for these two curves). $\mathrm{B}$, the $\mathrm{CO}$ model including $\mathrm{CK}-\mathrm{MB}$ and $\mathrm{PaO}_{2} / \mathrm{FiO}_{2}$ ratio shows satisfactory forecasting ability for DVT (AUC $=0.702 ; 95 \%$ Cl: 0.516 - 0.887; sensitivity: $58.8 \%$; specificity: $73.3 \%$ ) which has no significant difference compared with the DVT Wells score ( $P=0.242$ for these two curves) and the Padua prediction score ( $P=0.888$ for these two curves). $C$, the COD model including CK-MB, $\mathrm{PaO}_{2} / \mathrm{FiO}_{2}$ ratio, and $\mathrm{D}$-dimer level shows satisfactory forecasting ability for DVT (AUC $=0.803 ; 95 \% \mathrm{Cl}$ : 0.641 - 0.961; sensitivity: $66.7 \%$; specificity: $82.4 \%$ ) which significantly higher than that of the DVT Wells score ( $P=0.020$ for these two curves), and has no significant difference compared with the Padua prediction score ( $P=0.363$ for these two curves).

Abbreviations: $\mathrm{CO}=\mathrm{CK}-\mathrm{MB}+\mathrm{PaO}_{2} / \mathrm{FiO}_{2}$ ratio $\mathrm{COD}=\mathrm{CK}-\mathrm{MB}+\mathrm{PaO}_{2} / \mathrm{FiO}_{2}$ ratio + D-dimer level; $\mathrm{ARDS}$, acute respiratory distress syndrome; $\mathrm{AUC}$, area under the curve; $\mathrm{Cl}$, confidence interval; $\mathrm{CK}$, creatine kinase isoenzyme; DVT, deep vein thrombosis; $\mathrm{FiO}_{2}$, fraction of inspired oxygen; $\mathrm{PaO}_{2}$, partial pressure of arterial oxygen; ROC, receiver operating characteristic. 


\section{Figure 6}

Nomogram for screening for DVT of prediction variables.

The nomogram was draw based on the selected three predictors. The corresponding points can be obtained by making a vertical line upward based on the value of each variable. The total points can be obtained by adding the points of the three variables. The probability of survival (without DVT) in 5 days, 7 days and 14 days can be obtained by making a vertical line downward based on the total points.

\section{Supplementary Files}

This is a list of supplementary files associated with this preprint. Click to download.

- SupplementaryTable.docx

- SupplementaryFigure.pdf 